\title{
OS EQUINÓIDES PYGORHYNCHUS COLOMBIANUS (COOKE, 1955) E PSEUDHOLASTER ALTIUSCULUS (WHITE, 1887) NO NEOALBIANO DA BACIA DE SERGIPE, BRASIL
}

\author{
CYNTHIA LARA DE CASTRO MANSO \& WAGNER SOUZA-LIMA \\ Fundação Paleontológica Phoenix, Rua Geraldo Menezes de Carvalho, 218, 49050-750, Aracaju, SE, Brasil. \\ cynthia@phoenix.org.br,wagner@phoenix.org.br
}

\begin{abstract}
RESUMO - A espécie Pygorhynchus colombianus (Cooke, 1955) é assinalada pela primeira vez no Brasil, ocorrendo em depósitos cretácicos (Albiano superior) da bacia de Sergipe. Pseudholaster altiusculus (White, 1887) é também registrada pela primeira vez para o Albiano desta bacia. Pseudholaster altiusculus foi originalmente descrita como Toxaster altiusculus White, 1887, com base em espécimes coletados em rochas da bacia de Pernambuco-Paraíba. Neste trabalho é proposta a transferência desta espécie para o gênero Pseudholaster Pomel, 1883 (Ordem Holasteroida Durham \& Melville, 1957, Família Holasteridae Pictet, 1857) com base na disposição das placas do sistema apical. Evidências tafonômicas das carapaças sugerem que os equinóides foram preservados in situ ou sofreram pouco transporte.
\end{abstract}

Palavras-chave: Echinoidea, Cassiduloida, Holasteroida, bacia de Sergipe, Cretáceo.

\begin{abstract}
THE ECHINOIDS PYGORHYNCHUS COLOMBIANUS (COOKE, 1955) E PSEUDHOLASTER ALTIUSCULUS (WHITE, 1887) IN THE UPPER ALBIAN OF THE SERGIPE BASIN, BRAZIL. The echinoid species Pygorhynchus colombianus (Cooke, 1955) is recorded for the first time in the Cretaceous (Uppermost Albian) of the Sergipe Basin (NE Brazil). Pseudholaster altiusculus (White, 1887) is also referred for the first time to the Albian of this Basin. Pseudholaster altiusculus was originally described as Toxaster altiusculus White, 1887 based on specimens collected in the Pernambuco-Paraíba Basin (NE Brazil). In this paper is proposed to relocate T. altiusculus to the genus Pseudholaster Pomel, 1883 (Order Holasteroida Durhan \& Melville, 1957, Family Holasteridae Pictet, 1857), mainly based on the arrangement of the apical disc plates. Taphonomic data from the tests suggest that the echinoids were preserved in situ or underwent very little transport.
\end{abstract}

Key words: Echinoidea, Cassiduloida, Holasteroida, Sergipe Basin, Cretaceous.

\section{INTRODUÇÃO}

Entre as bacias marginais costeiras do leste brasileiro, a bacia de Sergipe possui uma das mais completas exposições de rochas relacionadas à evolução geológica do Atlântico Sul. Uma abundante e diversificada fauna é facilmente encontrada nestas rochas, destacando-se os amonóides e foraminíferos, que, a partir de sucessivos estudos bioestratigráficos (Bengtson, 1983; Koutsoukos \& Bengtson, 1993), têm permitido o refinamento da correlação das camadas aflorantes na bacia com a escala cronoestratigráfica internacional.

A bacia de Sergipe corresponde à parte sul do complexo conhecido como bacia Sergipe-Alagoas (Figura 1), que ocupa uma área de cerca de $20.000 \mathrm{~km}^{2}$ ao longo da faixa litorânea dos estados homônimos, no nordeste do Brasil. Seus depósitos compreendem rochas de idade neojurássica a pósmiocênicas, em geral fossilíferas. Os depósitos albianos, representados pela Formação Riachuelo, contêm uma variada fauna de equinóides, cuja distribuição reflete claramente as modificações e adaptações sofridas por este grupo desde a instalação de um ambiente marinho franco na área, provavelmente após o Neoaptiano.

A Formação Riachuelo está dividida em três membros: Maruim, Angico e Taquari (Feijó, 1995) (Figura 2). A deposição destes sedimentos transcorreu, provavelmente, do Neoaptiano até o Neoalbiano, quando uma grande transgressão, iniciada no Eocenomaniano, propiciou a deposição dos calcilutitos da Formação Cotinguiba. Os equinóides estudados foram coletados em rochas pertencentes ao Membro 


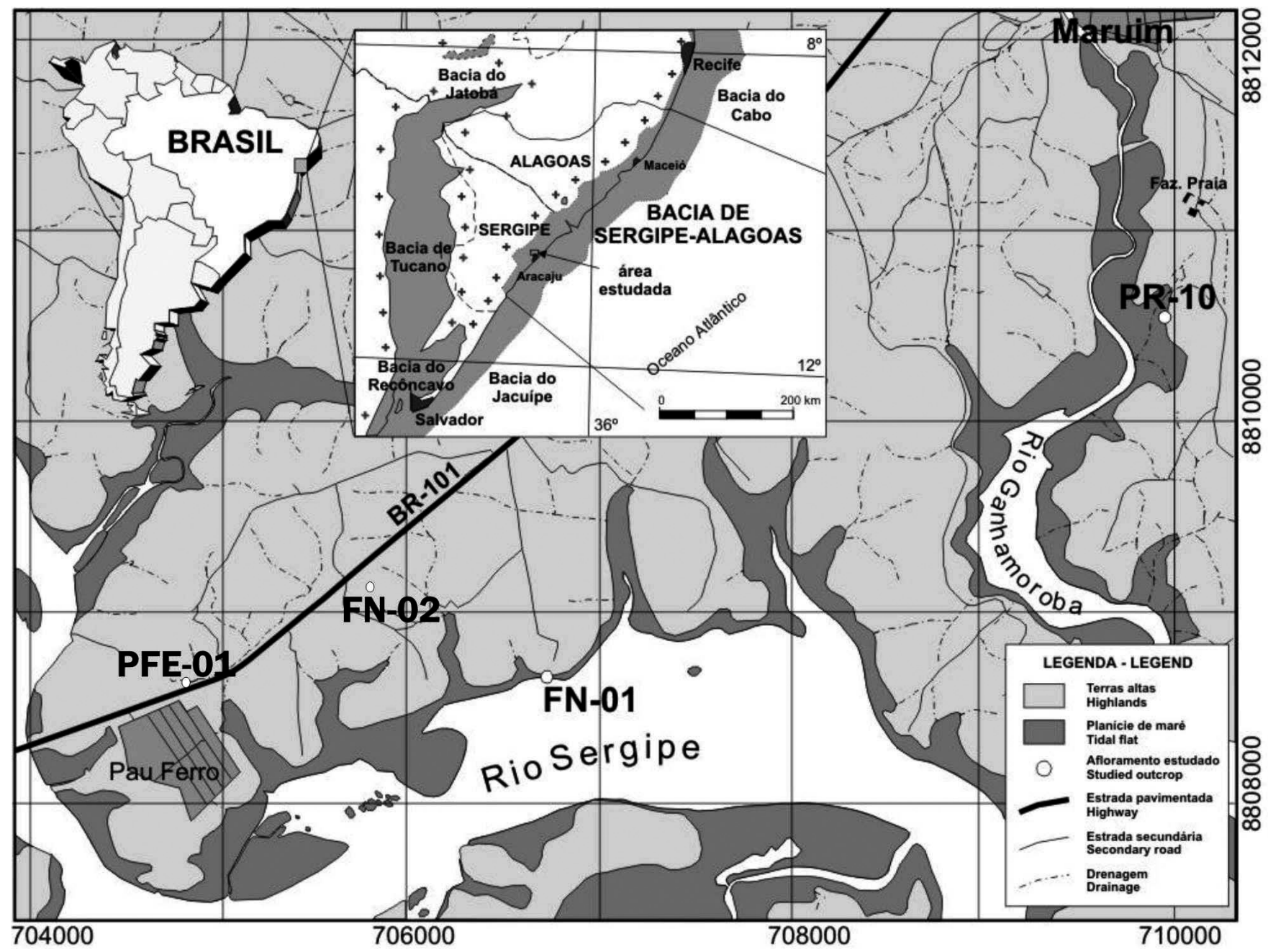

Figura 1. Mapa de localização dos afloramentos. PFE-01 = Pau Ferro 01; FN-01 = Fazenda Nova 01; FN-02 = Fazenda Nova $02 ;$ PR-10 = Praia 10

Figure 1. Location map of the outcrops. PFE-01 = Pau Ferro 01; FN-01 = Fazenda Nova 01; FN-02 = Fazenda Nova $02 ;$ PR-10 = Praia 10.

Maruim, posicionadas estratigraficamente na porção mais superior do Albiano superior pela ocorrência de amonóides do gênero Mortoniceras (Beurlen, 1961).

Durante o Cretáceo, houve um aumento no número de espécies de equinóides cassidulóides, cujo ápice ocorreu no Eoceno. Em seguida, ocorreu um decréscimo na diversidade, restando hoje pouco mais de uma dúzia de espécies (Kier, 1966). Assim como os cassidulóides, os holasteróides passaram por um período de maior diversificação durante o Cretáceo e Terciário. Atualmente, a maioria dos representantes habita as regiões mais profundas dos oceanos (Wagner \& Durham, 1966; Smith, 2004).

Nesse trabalho são assinaladas duas novas ocorrências de equinóides para o Albiano superior da bacia de Sergipe, o cassidulóide Pygorhynchus colombianus (Cooke, 1955) e o holasteróide Pseudholaster altiusculus (White, 1887). P. colombianus é registrado pela primeira vez para o Brasil, ao passo que Pseudholaster altiusculus ocorre na bacia de Pernambuco-Paraíba, tendo sido originalmente descrito como Toxaster altiusculus por White (1887).

\section{MATERIAL E MÉTODOS}

O material utilizado neste trabalho provém de coletas sistemáticas em rochas da Formação Riachuelo, depositado na coleção de invertebrados fósseis da Fundação Paleontológica Phoenix (FPH). Para comparação, foram examinados os materiais-tipo (lectótipo e paralectótipo) de Toxaster altiusculus White, 1887 depositados na coleção do Museu Nacional da Universidade Federal do Rio de Janeiro (MN), estudados e figurados por Brito (1981)

Após a preparação dos espécimes coletados, foram tomados os dados biométricos dos exemplares em melhor estado de preservação (Figura 3). As interpretações paleoecológicas basearam-se na análise morfológica de várias características da carapaça, segundo Kier (1962) e Smith (1984, 1995), e discutidas por Manso (2003).

\section{Localidades}

Os afloramentos estudados situam-se no Município de Maruim (Figura 1), concentrando-se próximos ou às mar- 


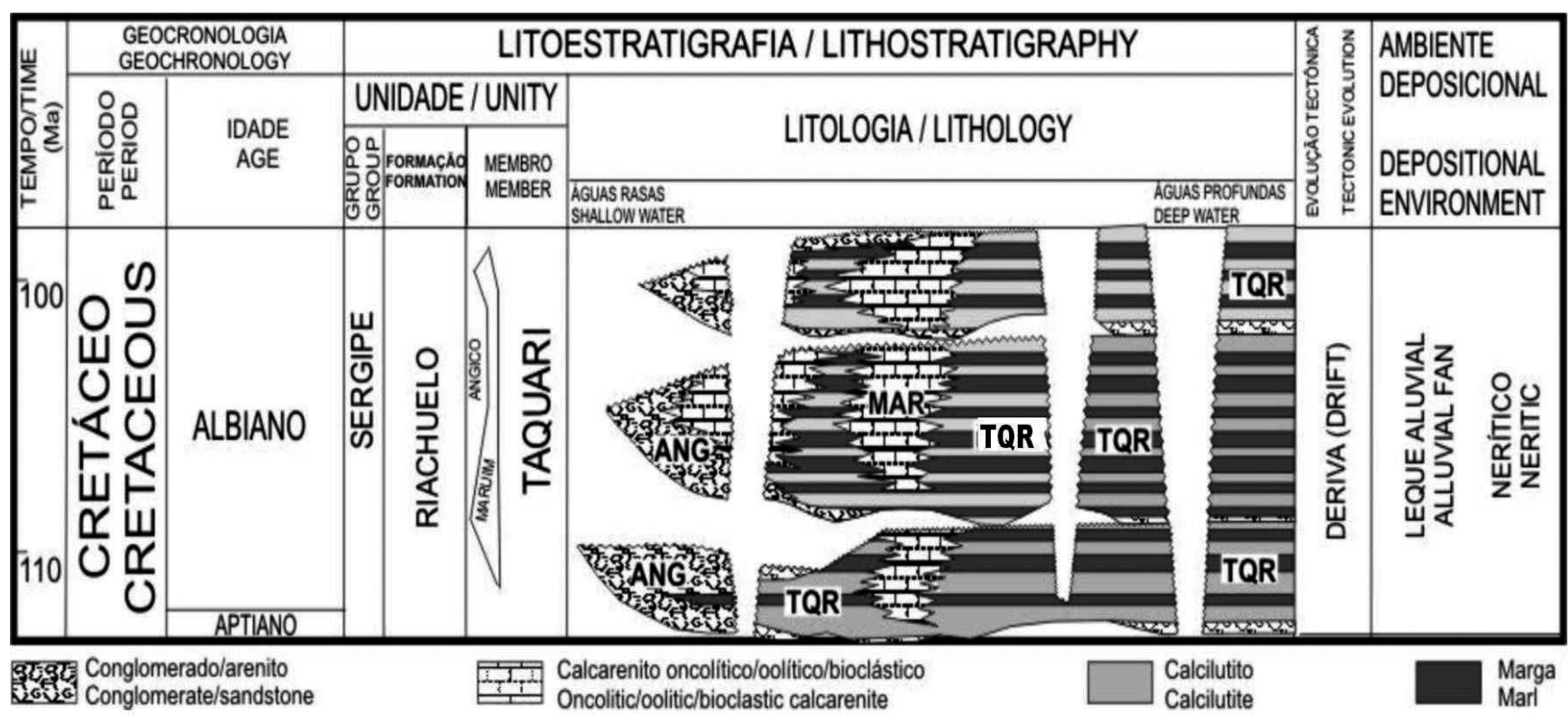

Figura 2. Carta estratigráfica da seção marinha aptiana-albiana da bacia de Sergipe (modificado de Souza-Lima et al., 2002). ANG = Membro Angico; MAR = Membro Maruim; TQR = Membro Taquari.

Figure 2. Stratigraphic chart of the marine Aptian-Albian section from the Sergipe Basin (modified from Souza-Lima et al., 2002). ANG = Angico Member; MAR = Maruim Member; TQR = Taquari Member.

gens do rio Sergipe, no trecho que antecede o encontro do rio Ganhamoroba, e na margem esquerda deste último rio, na planície de maré. São afloramentos de acesso relativamente fácil, dificultado apenas pelas marés altas ou durante a época das chuvas, quando alguns dos acessos (por exemplo, para Fazenda Nova 1) tornam-se praticamente intransitáveis. Estes afloramentos, em geral, caracterizam amplos lajedos ou blocos isolados, dificultando tanto a correlação entre eles quanto estudos bioestratigráficos.

Os afloramentos foram descritos utilizando-se a metodologia adotada por Bengtson (1983, p. 30-31). As coordenadas foram obtidas utilizando-se um GPS Ensign (TrimbleNavigation) sobre o datum Córrego Alegre, convertidas para o datum Aratu e arredondadas para os 50 metros mais próximos. As coordenadas UTM estão referenciadas ao meridiano central $39^{\circ}$. Os códigos em parênteses referem-se

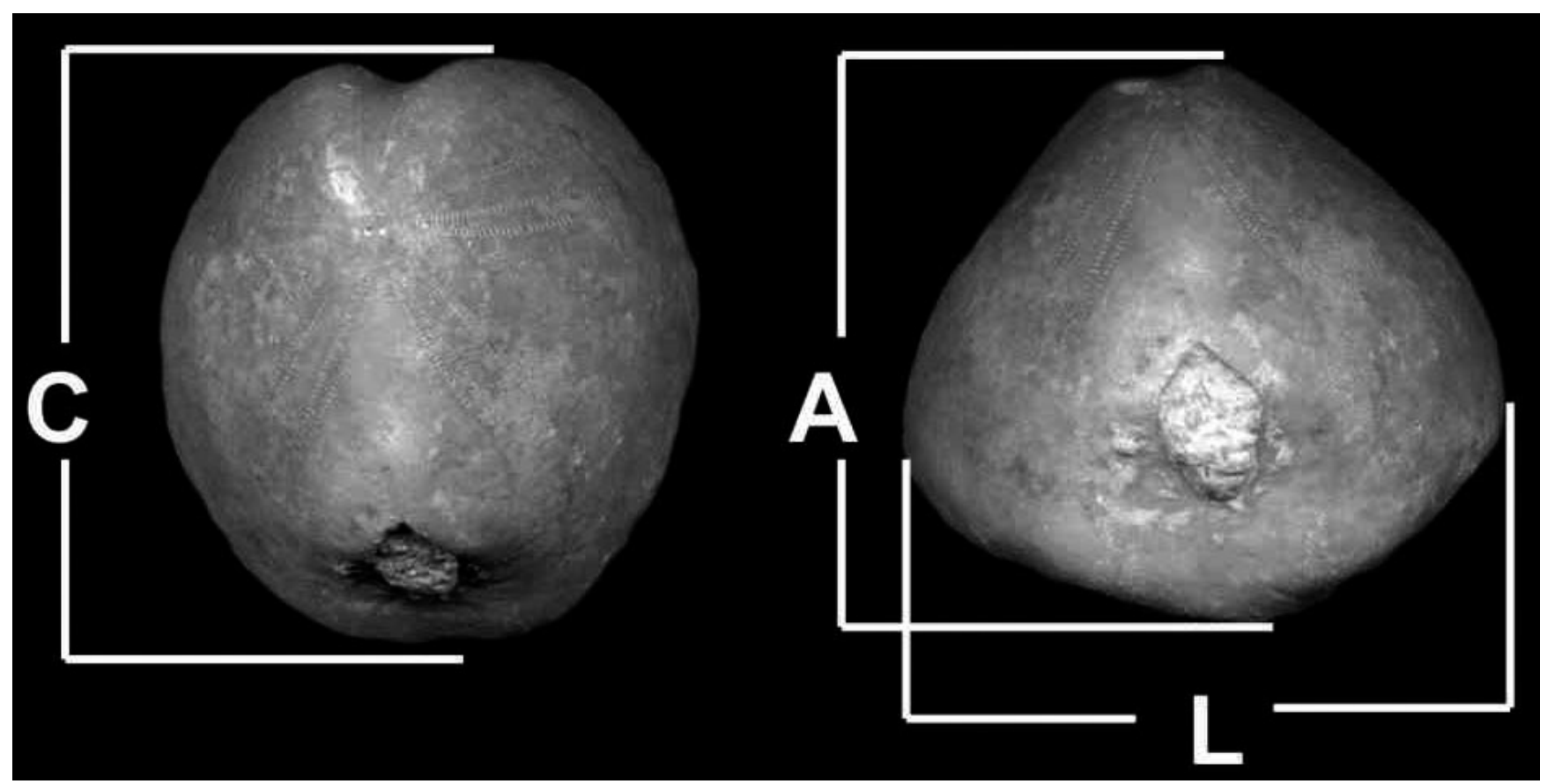

Figura 3. Medidas da carapaça: $A=$ altura; $C=$ comprimento; $L=$ largura.

Figure 3. Measurements of the test: $A=$ height; $C=$ length; $L=$ width. 
à identificação dos afloramentos no mapa de localização. O código Krm corresponde à Formação Riachuelo, Membro Maruim. Por exposição entende-se afloramento com altura inferior a $0,5 \mathrm{~m}$, enquanto seção representa afloramento com altura superior a $0,5 \mathrm{~m}$.

Fazenda Nova 1 (FN-01). UTM 8.808.650N/706.700E. Mapa topográfico folha: SC.24-Z-B-IV-4 Aracaju. Exposição na margem esquerda do rio Sergipe, cerca de $3 \mathrm{~km}$ a montante da confluência do rio Ganhamoroba, parcialmente coberta durante a maré alta. Altitude: $0 \mathrm{~m}$. Krm: calcarenito oncolítico/bioclástico, localmente bioturbado, com abundantes equinóides e amonóides. Referências: Manso \& Souza-Lima (2003).

Fazenda Nova 2 (FN-02). UTM 8.808.600N/706.200E. Mapa topográfico folha: SC.24-Z-B-IV-4 Aracaju. Seção exposta em pequena pedreira desativada, situada na margem esquerda do rio Sergipe, cerca de $1.200 \mathrm{~m}$ a montante do afloramento Fazenda Nova 1, com acesso a partir da BR 101. Altitude: $5 \mathrm{~m}$. $\mathrm{Krm}$ : calcarenito oncolítico/bioclástico, localmente bioturbado, com intercalações de calcarenitos argilosos contendo abundantes braquiópodes e equinóides.

Pau Ferro 1 (PFE-01). UTM 8.808.050N/705.050E. Mapa topográfico folha: SC.24-Z-B-IV-4 Aracaju. Pequena exposição em corpos isolados na borda do manguezal, no distrito de Pau Ferro, Maruim, parcialmente coberta durante a maré alta. Altitude: 0,5 m. Krm: calcarenito bioclástico/oncolítico creme-claro a cinza.

Praia 10 (PR-10). UTM 8.810.550N/709.950E; coordenadas geográficas $10^{\circ} 45^{\prime} 19^{\prime \prime S}, 37^{\circ} 04^{\prime} 44^{\prime \prime W}$. Mapa topográfico folha: SC.24-Z-B-IV-4 Aracaju. Pequena exposição em corpos isolados na planície de maré da margem esquerda do rio Ganhamoroba, na propriedade denominada Fazenda Praia, Maruim, parcialmente coberta durante a maré alta. Altitude: 0,5 m. Krm: calcarenito bioclástico/oncolítico creme claro a cinza, muito cimentado e calcarenito fino a médio creme escuro. Referências: corresponde à área denominada "Lastro" de White (1887), Branner (1890), Maury (1930, 1934, 1937), Brito (1980, 1981), e denominada Lastro ("Praia Area") de Bengtson (1983) e Smith \& Bengtson (1991); Praia 10 de Manso \& Souza-Lima (2003).

\section{SISTEMÁTICA PALEONTOLÓGICA}

\author{
Classe ECHINOIDEA Leske, 1778 \\ Ordem CASSIDULOIDEA Claus, 1880 \\ Família NUCLEOLITIDAE Agassiz \& Desor, 1847 \\ Gênero Pygorhynchus Agassiz, 1839
}

Pygorhynchus colombianus (Cooke, 1955)

Figuras 4A-D e 5A-C

\author{
Material. FPH-382-I, FPH-383-I, FPH-413-I a FPH-415-I, \\ FPH-422-I.
}

Procedência. Fazenda Nova 2.

\section{Descrição}

Carapaça. Contorno oval, mais estreita na região anterior (Figuras 4A e 5A, B). Carapaças com 45,5 a 65,0 mm de com-
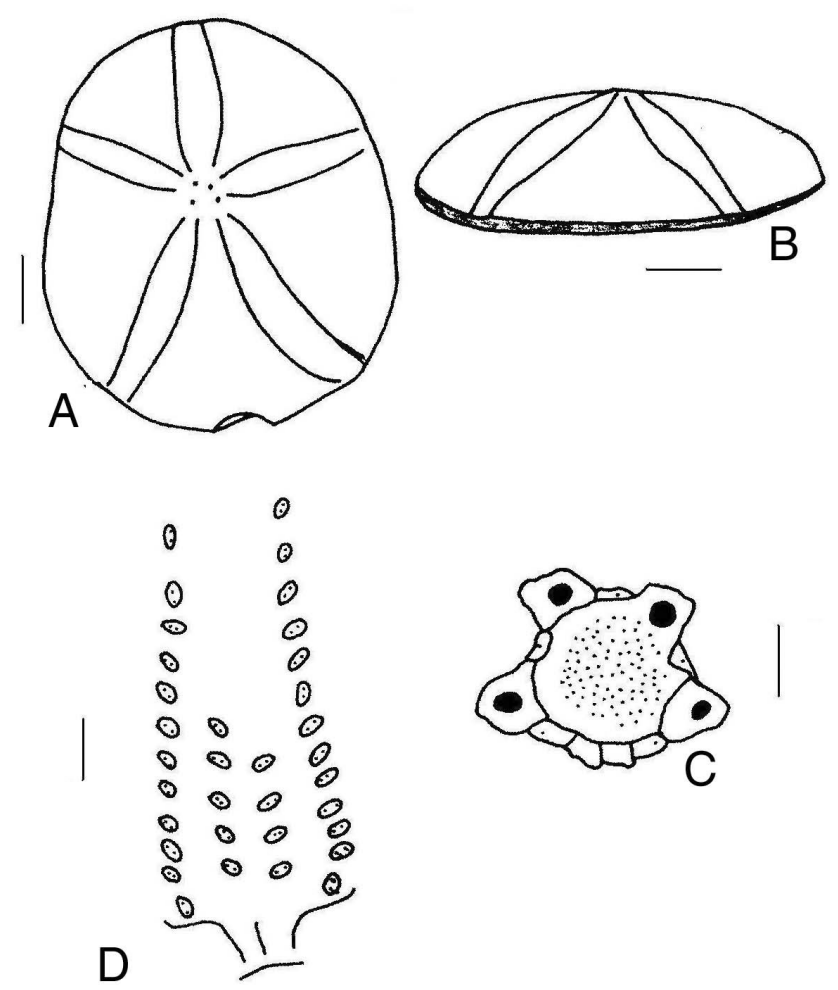

Figura 4. Desenho esquemático de Pygorhynchus colombianus (Cooke). A, vista aboral; B, vista lateral; C, placas do sistema apical; D, pares de poros dos filódios. Escala: A, B = $10 \mathrm{~mm}$; C, D $=1 \mathrm{~mm}$.

Figure 4. Schematic drawing of Pygorhynchus colombianus (Cooke). A, aboral view; B, lateral view; C, plates of apical system; $\mathbf{D}$, pores pairs of phyllodes. Scale bars: $\mathbf{A}, \mathbf{B}=10 \mathrm{~mm} ; \mathbf{C}, \mathbf{D}=1 \mathrm{~mm}$.

primento, 41,0 a 54,0 mm de largura e 12,5 e 23,5 mm de altura. O ponto mais alto da carapaça coincide com o sistema apical, que ocupa a parte central da região aboral, aproximadamente. A superfície inferior é plana, com o peristômio localizado quase em posição central.

Região apical. Sistema apical tetrabasal com a placa genital 2 (madreporita) muito maior do que as demais. Placas oculares menores que as genitais e bem separadas (Figura 4C).

Região ambulacral. Todos os cinco ambulacra petalóides e todas as pétalas abertas distalmente. Pétalas com 9,0 mm de largura máxima, estendendo-se até próximo à região ambital (Figura 4A). As cinco pétalas possuem pares de poros conjugados, com os poros externos de cada coluna em forma de fenda diagonalmente alongada e os internos arredondados. Um par de poros em cada placa ambulacral, além das pétalas. Região interambulacral. Recoberta por pequeninos tubérculos, maiores na superfície oral.

Periprocto. De contorno oval, está situado lateralmente na extremidade posterior (Figura 5C). Esta estrutura mede 8,0 $\mathrm{mm}$ de comprimento por $6,5 \mathrm{~mm}$ de largura no exemplar FPH$383-\mathrm{I}$ (comprimento $=62,0 \mathrm{~mm}$ ) e está localizada próxima à base no maior exemplar (FPH-383-I) e um pouco mais alto no menor exemplar (FPH-415-I). Logo abaixo do periprocto, pode ser observado um sulco raso na direção ventral. Este sulco é mais acentuado no exemplar FPH-383-I. Nos exemplares exa- 


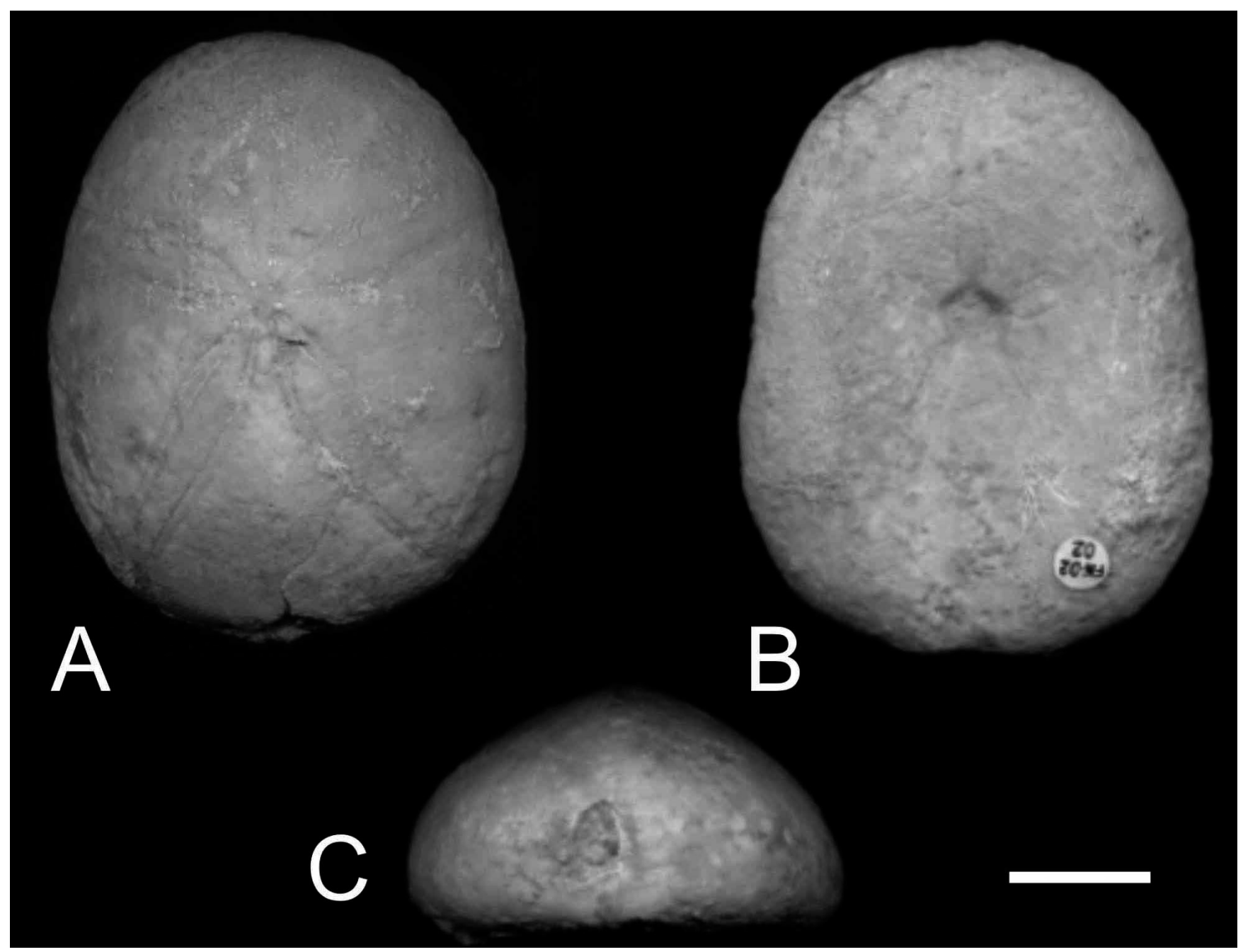

Figura 5. Pygorhynchus colombianus (Cooke), FPH-382-I: A,vista aboral; B, vista oral. FPH-383-I: C, vista posterior. Escala $=10 \mathrm{~mm}$. Figure 5. Pygorhynchus colombianus (Cooke), FPH-382-I: A, aboral view; $\mathbf{B}$, oral view. FPH-383-I: C, posterior view. Scale bar $=10 \mathrm{~mm}$

minados, o periprocto pode ser visto tanto da parte dorsal quanto da parte ventral da carapaça.

Perístoma. Semipentagonal, situado próximo ao centro e sem poros bucais. Filódios do tipo nucleolitídio, formados por uma série externa de mais de 13 pares de poros e uma série interna de seis a sete pares de poros (Figura 4D). Os tubérculos próximos ao perístoma (bourrelets) são fracamente desenvolvidos.

\section{Discussão}

Os exemplares descritos evidenciam, como características principais, a ausência de poros bucais e a presença de dois pares de poros por placa, além de pétalas. Kier (1962) transferiu Arnaudaster colombianus Cooke para o gênero Pygorhynchus Agassiz ao perceber que os exemplares descritos por Cooke (1955) possuíam as mesmas características. Os exemplares de Sergipe diferem daqueles revisados por Kier (1962) apenas na largura da carapaça, que é mais estreita (entre 41,0 e 54,0 mm), provavelmente uma variação ontogenética.
Smith (in Smith \& Bengtson, 1991) refere-se a apenas três gêneros de cassidulóides que possuem carapaça deprimida e periprocto posicionados verticalmente no ambitus, de tal forma que este aparece como um entalhe posterior em ambas as faces dorsal e ventral. São eles: Pygorhynchus, Arnaudaster e Parapygus. Os gêneros Arnaudaster e Parapygus pertencem à família Echinolampadidae Gray, 1851, e possuem poros bucais; o gênero Pygorhynchus, classificado na família Nucleolitidae Agassiz \& Desor, 1847, não apresenta poros bucais (Kier, 1962). Além disso, Pygorhynchus possui dois poros nos filódios em cada placa, diferenciando-se assim de Parapygus e Arnaudaster.

\section{Distribuição estratigráfica e geográfica}

O gênero Pygorhynchus foi descrito do Neocomiano ao Albiano da Europa, África e Américas. Segundo Smith \& Bengtson (1991), Pygorhynchus é conhecido do Aptiano do Peru e Venezuela, e sua ocorrência não ultrapassa o Albiano. De acordo com Kier (1962), durante e após o Cenomaniano, as espécies com filódios nucleolitídeos tiveram o número de 
poros dos filódios de cada placa reduzidos de dois para um, com aparecimento dos poros bucais. Desta forma $P$. colombianus poderia ser utilizado como um complemento auxiliar na bioestratigrafia do Albiano na bacia de Sergipe. $P$. colombianus é conhecido do Albiano superior da região de Vale Rancheria (Colômbia), sendo agora definida a sua ocorrência na parte final do Albiano superior do Membro Maruim da Formação Riachuelo, bacia de Sergipe, Brasil.

\section{Ordem HOLASTEROIDA Durham \& Melville, 1957 \\ Família HOLASTERIDAE Pictet, 1857 \\ Gênero Pseudholaster Pomel, 1883}

\section{Pseudholaster altiusculus (White, 1887) Figuras 6A-D e 7A-D}

1887 Toxaster altiusculus White, White, p. 262, est. 28, figs. 9-13.

1981 Toxaster altiusculus. Brito, p. 569, est. 1, figs. 3-5. 1991 Toxaster altisulcus. (error) Smith (in Smith \& Bengtson, 1991), p. 50.

\section{Material examinado}

Materiais-tipo. Toxaster altiusculus, MN 3088-I, lectótipo; MN 3089-I, paralectótipo

Materiais não-tipos. FPH-343-I; FPH-345-I; FPH-348-I a FPH354-I; FPH-357-I a FPH-363-I; FPH-416-I; FPH-417-I; FPH419-I; FPH-587-I; FPH-549-I; FPH-569-I; FPH-587-I; FPH-632I; FPH-635-I; FPH-637-I.

Procedência. Fazenda Nova 1, Fazenda Nova 2, Pau Ferro 1 e Praia 10.

Diagnose emendada. Espécie de tamanho médio, com a região dorsal elevada, semicônica; com sulco anterior profundo; sistema apical subcentral; ambulacros não petalóides; região anterior arredondada e proeminente; periprocto oval situado longitudinalmente no alto da região posterior; perístoma oval situado transversalmente na região anterior.

\section{Descrição}

Carapaça. Variando entre 17,0 e 40,5 mm de comprimento, altura entre $68 \%$ e $72 \%$ do comprimento. Carapaça de contorno cordiforme, cujo ponto mais alto coincide com o sistema apical. Observado lateralmente, nota-se um declive que vai do sistema apical ao periprocto (Figuras 6A e 7B).

Região apical. $\mathrm{O}$ disco apical mostra o típico arranjo das placas dos holasteróides e está posicionado anterior ao centro. Placas oculares II e IV separadas pela placa madreporita no maior exemplar, enquanto nos menores estas placas encontram-se juntas (Figuras 6C e 6D). Placa genital 3 separada das placas 1 e 4 . Madreporita com muitos poros ocupando boa parte do centro do sistema apical no maior exemplar (Figura 6C), não ocorrendo desta forma nos menores.

Região ambulacral. O ambulacro anterior estende-se do sistema apical ao perístoma, e possui os pares de poros não conjugados, de formato ligeiramente oval a arredondado. Os ambulacros pares (pétalas) não são sulcados, ocupando apro-
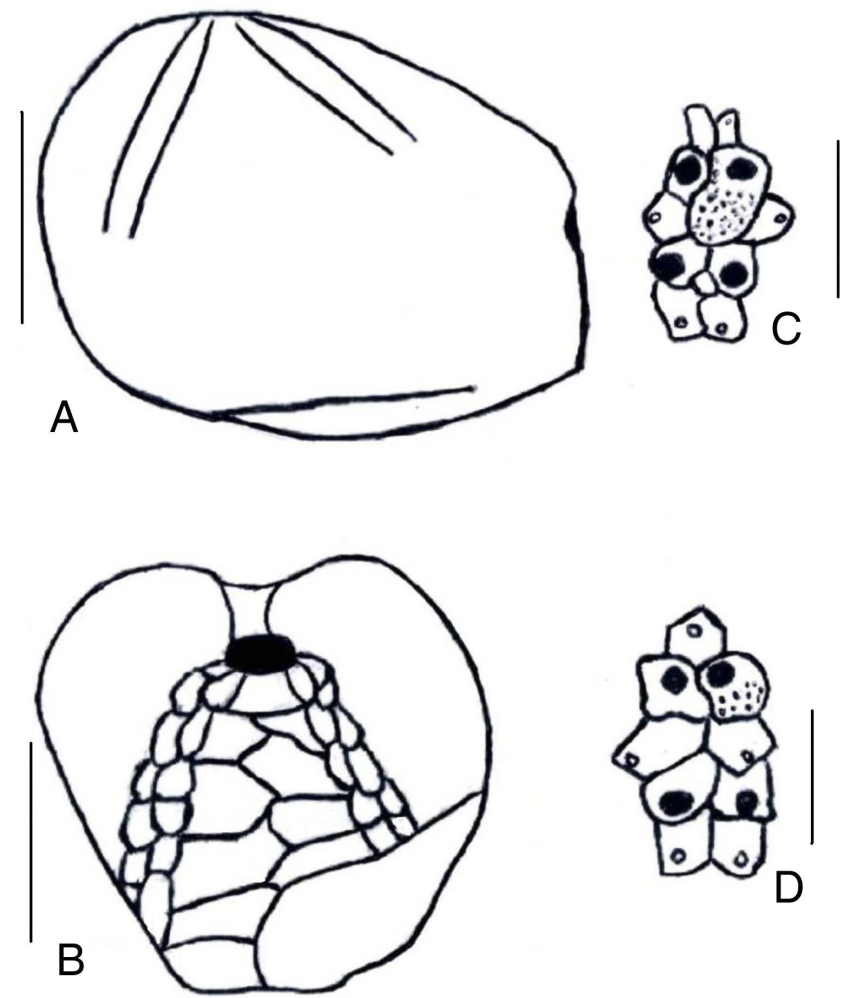

Figura 6. Desenho esquemático de Pseudholaster altiusculus (White). A, vista lateral; B, vista ventral; C-D, placas do sistema apical. Escalas: $\mathbf{A}, \mathbf{B}=10 \mathrm{~mm} ; \mathbf{C}, \mathbf{D}=1 \mathrm{~mm}$.

Figure 6. Schematic drawing of Pseudholaster altiusculus (White). A, lateral view; B, oral view; C-D, plates of apical system. Scale bars: $\mathbf{A}, \mathbf{B}=10 \mathrm{~mm} ; \mathbf{C}, \mathbf{D}=1 \mathrm{~mm}$.

ximadamente $80 \%$ da altura da carapaça. Podem ser observadas diferenças entre as colunas de poros tanto nas pétalas II e IV quanto nas pétalas I e V. A coluna anterior é inicialmente formada por poros pequenos e arredondados, um pouco maiores no exemplar FPH-348-I, e razoavelmente maiores nos demais exemplares, quanto maior for a distância do sistema apical. Os três últimos poros são pequeninos e arredondados como os iniciais. A coluna posterior apresenta os primeiros três poros próximos ao sistema apical, de tamanho pequeno. Em direção ao meio da pétala, tornam-se maiores e em forma de fenda. Diminuem em seguida até tornarem-se pequenos (aproximadamente os três últimos pares) e do mesmo tamanho dos pares da coluna anterior. Pétalas abertas. Pétalas I e V semelhantes às II e IV. Apesar desta semelhança, os poros das colunas das pétalas posteriores são pouco menores que os observados nestas mesmas colunas, nas pétalas II e IV.

Região interambulacral. Com a tuberculação aboral mostrando poucos tubérculos maiores espalhados irregularmente sobre a carapaça, com maior concentração na região anterior próximo ao ambulacro III, na região ventrolateral e na região do plastrão. Pequeninos grânulos aparecem por toda a carapaça, mostrando que ela era recoberta principalmente por pequenos espinhos.

Periprocto. Abertura de formato oval situada logo acima do ambitus (Figura 7A) e com, aproximadamente, o mesmo tama- 


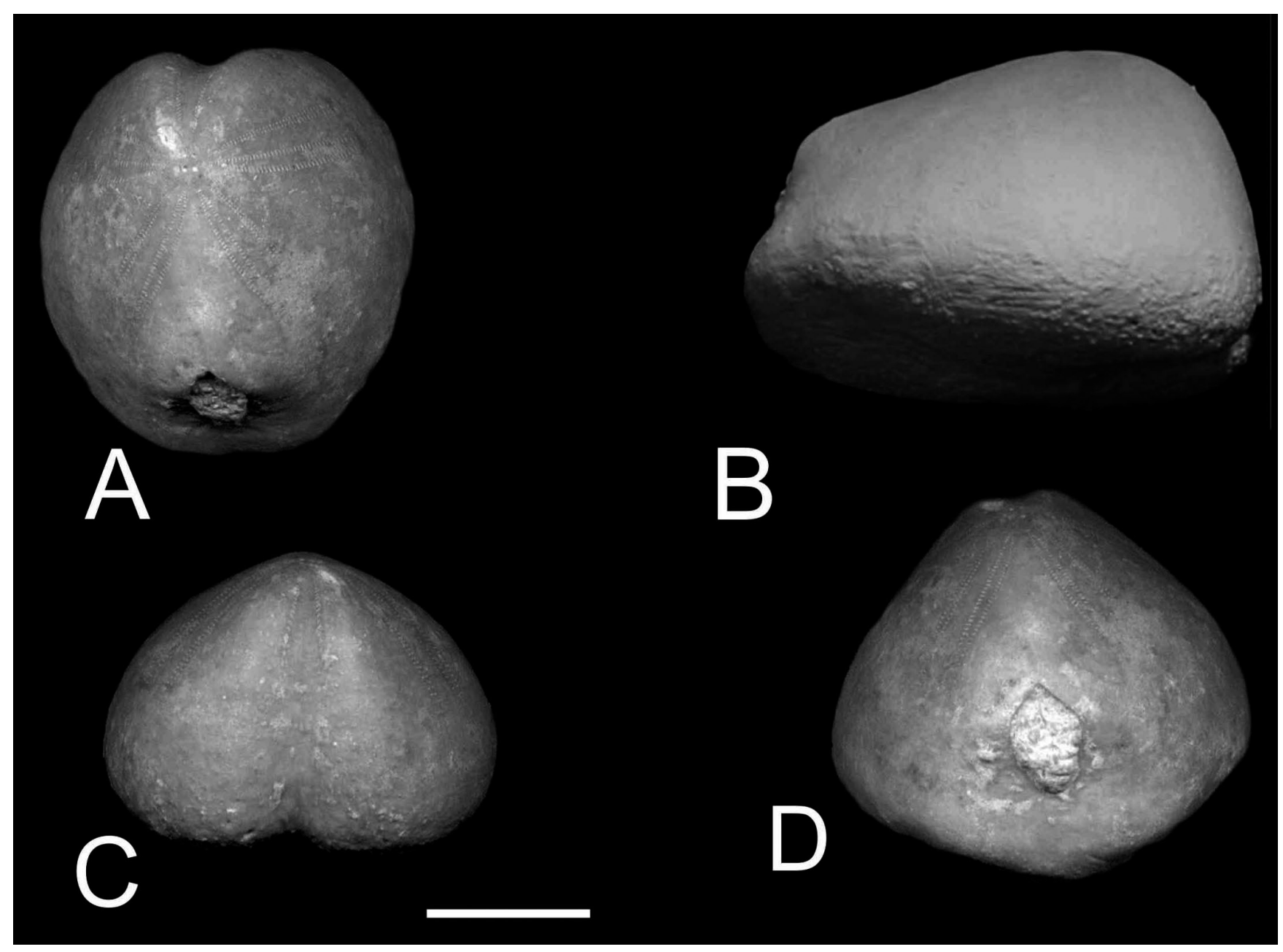

Figura 7. Pseudholaster altiusculus (White), FPH-349-I: A, vista aboral; B, vista lateral; C, vista anterior; D, vista posterior. Escala = $10 \mathrm{~mm}$ Figure 7. Pseudholaster altiusculus (White), FPH-349-I: A, aboral view; B, lateral view; C, anterior view; $\mathbf{D}$, posterior view. Scale bar = $10 \mathrm{~mm}$.

nho do peristômio. Um sulco raso pode ser observado abaixo do periprocto e se prolonga em direção à região ventral. Em alguns exemplares na região infralateral do periprocto podem ser observados alguns pares de poros.

Perístoma. Relativamente grande, com $3,5 \mathrm{~mm}$ de comprimento no exemplar FPH-343-I, sulcada com uma leve projeção de labrum.

Plastrão. Protosterno com uma quilha mediana (Figura 6B). Variações intraespecíficas. Alguns exemplares, principalmente aqueles da localidade Pau-Ferro 01, apresentaram acentuada compressão lateral, sendo seus dados biométricos desprezados. A maioria dos exemplares mostrou que a razão entre largura e comprimento esteve situada entre 0,91 e 0,95 , e a razão entre altura e comprimento entre 0,66 e 0,7 , não tendo sido considerado significativo. Foram também observadas modificações no tamanho e disposição da placa genital 2, estando no maior exemplar bastante desenvolvida e separando as placas oculares II e IV, enquanto nos menores exemplares possui o tamanho aproximado das demais placas apicais e não se interpõe entre as placas oculares. Variações no tamanho e no número de placas genitais anteriores nos holasteróides podem ser assumidas como variações ontogenéticas (Smith, 2004).

\section{Discussão}

A análise dos exemplares estudados permite incluí-los na espécie originalmente descrita por White (1887) como Toxaster altiusculus, com base em espécimes provenientes de rochas do Cretáceo superior do Estado de Pernambuco. Entretanto, o arranjo das placas do sistema apical, é típico dos holasteróides, justificando sua transferência do gênero Toxaster Agassiz (ordem Spatangoida Claus, 1876) para o gênero Pseudholaster Pomel (ordem Holasteroida Durham \& Melville, 1957). Esta espécie é semelhante em muitos aspectos a Pseudholaster tricarinatus Lambert, 1924, descrita e figurada por Smith (in Smith \& Bengtson, 1991, p. 50) para a parte mais superior do Albiano superior e Cenomaniano inferior de Sergipe. $P$. altiusculus difere de $P$. tricarinatus no formato da carapaça, mais cônica em $P$. altiusculus, e no ambulacro III, localizado no sulco que segue em direção ao perístoma. Estas também são as principais características que diferenciam Pseudholaster altiusculus de $P$. androaviensis 
(Lambert, 1933) do Albiano de Madagascar e de Holaster lerichei Dartavelle, 1953 do Albiano superior de Angola (África). Smith (in Smith \& Bengtson, 1991) sugere que Holaster lerichei e $P$. androaviensis seriam sinônimas de Pseudholaster tricarinatus Lambert. Pseudholaster altiusculus também se assemelha a $P$. vanhoepeni (Besaire \& Lambert, 1930), do Albiano superior da bacia de Zuzuland (África do Sul), diferindo principalmente na altura da carapaça - mais alta em P. altiusculus - e por possuir uma quilha mais pronunciada no plastrão e o sulco anterior em posição mais vertical.

\section{Distribuição estratigráfica e geográfica}

O material descrito por White (1887) foi referido às localidades de Maria Farinha e Igarassu, em Pernambuco e, provavelmente, à região de João Pessoa, na Paraíba. Tais localidades correspondem a regiões onde afloram os depósitos marinhos das formações Maria Farinha, de idade terciária (localidade de Maria Farinha) e Gramame, de idade campanianamaastrichtiana (demais localidades). Posteriormente, esta ocorrência foi citada equivocadamente por Brito (1981) como relativa a "Igarassu, Estado de Pernambuco(?), Formação Maria Farinha, Paleoceno". Este é o primeiro registro de $P$. altiusculus na bacia de Sergipe.

\section{PALEOECOLOGIA}

A presença de pares de poros em todas as placas ambulacrais leva à suposição de que, em Pygorhynchus colombianus, os pódios tenham tido função respiratória. As pétalas estendidas até quase a região ambital e a carapaça com a face ventral plana e a dorsal levemente convexa, sugerem um modo de vida semi-endofaunal, pois, ao contrário dos espatangóides, estes animais não possuíam fascíolos nem pódios especializados no ambulacro anterior que possibilitasse uma sobrevivência totalmente endofaunal (Kier, 1962). Possivelmente, esta espécie viveu em ambiente de plataforma, abaixo da linha da maré, na base das ondas normais, entre 5-20 m de profundidade, mas distante da margem e em ambiente relativamente protegido. Muito provavelmente, o substrato seria inconsolidado e altamente permeável, composto por areia média a grossa, e com pouca contribuição de partículas mais finas. Estas inferências baseam-se em cassidulóides do gênero Echinolampas, que possuem o formato da carapaça muito semelhante à de Pygorhynchus e é encontrado em mares atuais (Smith, 1995). Apesar da biologia das espécies recentes de Echinolampas serem pobremente documentada, Thum \& Allen (1975) indicam a preferência destes organismos por viverem no interior de substratos formados por areia grossa e conchas, em regiões pouco profundas. Pesquisas paleoecológicas sobre espécies fósseis de Echinolampas sugerem, ainda, que estes organismos viveram em regiões com profundidade de água entre 10 e 500 onde habitariam fundos arenosos e viveriam parcialmente enterrados até as extremidades das pétalas (Kroh \& Nebelsick, 2003). A associação de P. colombianus com Coenholectypus neocomiensis (Gras, 1848) sugere sua ocor- rência próxima a construções recifais (Néraudeau et al., 1993). Evidências diretas destas construções não foram encontradas, porém alguns afloramentos próximos apresentam pólipos de corais isolados (p. ex., Praia 10) e bioclastos atribuídos a algas vermelhas solenoporáceas. A presença de um grande periprocto e de um sulco anal, relacionado a uma alta produção fecal, sugere que este equinóide era capaz de ingerir grandes quantidades de sedimento junto com as partículas alimentares (Smith, 1995; Kier, 1962). Junto a esta espécie ocorrem também os equinóides Temnocidaris (Stereocidaris) malheiroi (Loriol, 1888), Tetragramma deshayesi (Cotteau, 1864) e Pseudholaster altiusculus (White, 1887).

Pseudholaster altiusculus aparentemente viveu semienterrado em ambientes próximos àqueles em que Pygorhynchus colombianus teria vivido, porém numa fração sedimentar mais fina. Provavelmente, teria sido detritívoro seletivo como outros holasteróides (Smith \& Jeffery, 1998), devido à presença de pódios bucais. Junto a esta espécie foram coletados também os equinóides Phalacrocidaris branneri (White, 1887), Temnocidaris (Stereocidaris) malheiroi (Loriol, 1888), Tetragramma deshayesi (Cotteau, 1864), Coenholectypus neocomiensis (Gras, 1848), Phyllobrissus freitasii (White, 1887), Pygorhynchus colombianus (Cooke, 1955), Pseudholaster tricarinatus Lambert, 1924, e Hemiaster zululandensis Besairie \& Lambert, 1930. Na localidade Fazenda Nova 1, Pseudholaster altiusculus constitui um dos fósseis mais abundantes, sendo sobrepujado apenas pela espécie $H$. zululandensis Besairie \& Lambert, 1930 (Manso \& Souza-Lima, 2003).

Apesar dos equinóides terem sido coletados em sedimentos traduzidos como de alta energia, foram encontrados em sua grande maioria inteiros, com boa preservação. As relações tafonômicas e principalmente o hábito semiinfaunal e/ou infaunal, podem explicar a presença destes espécimes em posição de vida naquelas localidades. Desta forma, a relativa fragilidade da carapaça destas espécies sugere que estes organismos formam um registro autóctone e foram preservados aparentemente in situ, tendo sido submetidos a pouco ou nenhum transporte. Esta assembléia poderia ser classificada como "Concentração primariamente biogênica" (Fürsich \& Oschmann,1993 in Simões \& Holz, 2004), onde uma concentração de bioclastos possuindo uma alta porcentagem de fósseis preservados em posição de vida, incluindo invertebrados da epifauna como braquiópodes (presentes na localidade Fazenda Nova 2), indicam pouco ou nenhum distúrbio de fundo, durante a gênese desta concentração.

\section{CONSIDERAÇÕES FINAIS}

De um modo geral, os holasteróides se diversificaram a partir de um número reduzido de linhagens no início do Cretáceo, se expandiram no Cenomaniano e atingiram o máximo de diversidade no Maastrichtiano (Smith, 2004). O táxon Pseudholaster ocorre do Cretáceo inferior ao superior, tendo sido um dos membros mais primitivos do grupo Cardiasterina Pomel por apresentar plastrão protosterno, ausência de 
fascíolos e hidroporos confinados à placa genital 2 (Smith, 2004). A extinção dos Pseudholaster - como a da maioria dos holasteróides adaptados a águas mais rasas de plataforma interna - ocorreu durante o evento global que modificou as condições oceanográficas, no final do Cretáceo (Smith, 2004). No Terciário, as linhagens de holasteróides sobreviventes eram em sua maioria formadas por táxons de água profunda e que desenvolveram um modo mais especializado de alimentação, coletando detritos orgânicos floculados da interface água-sedimento (Jeffery, 2001).

A revisão dos materiais-tipo de Toxaster altiusculus White, 1887 permitiu incluí-lo no gênero Pseudholaster Pomel, 1883 (Ordem Holasteroida Durhan \& Melville, 1957, Família Holasteridae Pictet, 1857), uma vez que os espécimes revisados possuem características morfológicas específicas da ordem Holasteroida. Este estudo marca também o primeiro registro da espécie Pygorhynchus colombianus (Cooke, 1955) no Brasil e da espécie Pseudholaster altiusculus (White, 1887) na bacia de Sergipe.

As relações tafonômicas e o hábito semi-infaunal e/ou infaunal sugerem preservação dos equinóides in situ e em posição de vida, apesar de terem sido coletados em sedimentos traduzidos como de alta energia. A presença de braquiópodes em uma das localidades denuncia pouco ou nenhuma perturbação do fundo, durante a diagênese.

\section{AGRADECIMENTOS}

Nossos agradecimentos a Antônio Carlos S. Fernandes (MN/UFRJ), pelas facilidades oferecidas ao exame dos tipos estudados e a Rita de Cássia T. Cassab (Departamento Nacional de Produção Mineral), pelo auxílio no acesso à bibliografia. A Facelúcia B. C. Souza (UFBA) e aos revisores, pelas críticas e sugestões.

\section{REFERÊNCIAS}

Agassiz, L. 1839. Description des échinodermes fossiles de la Suisse; Première partie: Spatangoïdes et Clypéastroïdes. Mémoires de la Societé Héveltique de Sciences Naturelles, 3:1-101.

Agassiz, L. 1840. Catalogus systematicus ectyporum echinodermatum fossilium musei Necomiensis. Petitpierre, Neuchâtel, 20 p.

Agassiz, L. \& Desor, E. 1846-1947. Catalogue raisonné des familles, dés genres et dês especès de la classe de Échinodermes: Echinides. Annales des Sciences Naturelles et Zoologie. 3ème série, 6:305374; 7:129-168; 8:5-35, 355-380.

Bengtson, P. 1983. The Cenomanian-Coniacian of Sergipe Basin, Brazil. Fossils and Strata, 12:1-78.

Besairie, H. \& Lambert, J. 1930. Notes sur quelques échinides de Madagascar et du Zululand. Bulletin de la Societé Géologique de France, 30(4):107-117.

Beurlen, K. 1961. Die Kreide im Küstenbereich von Sergipe bis Paraiba do Norte (Brasilien). Zeitschrift der Deutschen Geologischen Gesellschaft, 112(3)[Jahrgang 1960]:378-384.

Branner, J.C. 1890. The Cretaceous and Tertiary Geology of the Sergipe-Alagoas Basin of Brazil. Transaction of the Ameican Philosophical Society. 16:369-434.

Brito, I.M. 1980. Os Equinóides fósseis do Brasil: I. Os endocíclicos.
Anais da Academia Brasileira de Ciências, 52(3):569-590.

Brito, I.M. 1981. Os equinóides fósseis do Brasil: III. Espatangoides, exceto Hemiasteridae. Anais da Academia Brasileira de Ciências, 53(3):569-578.

Claus, C.F.W. 1876-1880. Grundzüge der Zoologie. $3^{\text {rd }}$ ed., Marburg \& Leipzig, $2.522 \mathrm{p}$.

Cooke, C.W. 1955. Some Cretaceous echinoids from the Americas. United States Geological Survey, Professional Paper, 264-E:87112.

Cotteau, G. 1861-67. Paléontologie Française: Terrains Crétacés, 7 (Echinides). V. Masson, Paris. 892 p.

Dartevelle, E. 1953. Echinides fossiles du Congo et de l'Angola. Part 2: Description systématique dês Echinides fossiles du Congo et de 1'Angola. Annales du Musée Royal du Congo Belge, Tervuren, Série 8, 13:240.

Durham, J.W. \& Melville, R.V. 1957. Classification of Echinoids. Journal of Paleontology, 31(1):242-272.

Feijó, F.J. 1995. Bacias de Sergipe e Alagoas. Boletim de Geociências da Petrobras, 8 [para 1994]:149- 161.

Gras, C.J.A. 1848. Description des oursins fossiles du Département de l'Isère. Bulletin de la Société de Statistique des Sciences Naturelles et des Arts Industriels du Départament de L'Isère, Premiére Série, 4:293-381.

Gray, J.R. 1851. Description of some new genera and species of Spatangidae in the British Museum. The annals \& Magazine of Natural History, Second series, 3:130-134.

Jeffery, C.H. 2001. Heart urchins at the Cretaceous/Tertiary boundary: a tale of two clades. Paleobiology, 27:140-158.

Kier, P.M. 1962. Revision of the cassiduloid echinoids. Smithsonian Miscelaneous Collections, 144:1-262.

Kier, P.M. 1966. Cassiduloids. In: R.C. Moore (ed) Treatise on Invertebrate Paleontology, Part U Echinodermata. Geological Society of America, University of Kansas, 3, p. U492-U523.

Koutsoukos, E.A.M. \& Bengtson, P. 1993. Towards an integrated biostratigraphy of the Upper Aptian-Maastrichtian of the Sergipe-Basin, Brazil. Document Laboratoire Géologique de Lion, 125:241-262.

Kroh, A. \& Nebelsick J.H. 2003. Echinoid assemblages as a tool for palaeoenvironmental reconstruction - an exemple from the Early Miocene of Egypt. Palaeogeography, Palaeoclimatology, Palaeoecology, 201:157-177.

Lambert, J., 1920. Étude sur quelques formes primitives de Spatangides. Bulletim de la Societé de Science et Histoire. Naturelle de L'Yonne, 73:107-147.

Lambert, J. 1924. Pseudholaster. In: J. Lambert \& P. Thiéry. 19091925 (eds.) Essai de nomenclature raisonnés des échinides. Libraire Ferriére (Chaumont), 607 p.

Lambert, J. 1933. Echinides de Madagascar communiqués par M.H. Besairie.Annales géologiques du Service dês Mines, Madagascar, 3:7-49.

Leske, N.G. 1778. Jacobi Theodori Klein naturalis dispositio Echinodermatum. Edita et descriptionibus novisque inventis et synonymis auctorum aucta, Leipzig, $278 \mathrm{p}$.

Loriol, P. 1888. Description des échinides. In: P. Choffat \& P. de Loriol (eds.) Matériaux pour l'étude stratigraphique et paléontologique de la Province de l'Angola. Mémoires de la Société de Physique et d'Histoire, Naturelle de Genève, 30(2):97-114.

Manso. C.L.C. 2003. Paleoecologia, Paleobiogeografia e Sistemática dos Equinóides do Aptiano-Albiano (Cretáceo) do Brasil. Programa de Pós-graduação em Geologia, Universidade Federal da Bahia, Tese de Doutorado, 206 p.

Manso. C.L.C. \& Souza-Lima, W. 2003. O registro do equinóide 
Hemiaster zululandensis Besaire \& Lambert, 1930 no Cretáceo (Albiano Superior) de Sergipe. Revista Brasileira de Paleontologia 6:61-67.

Maury, C.J. 1930. O Cretaceo da Parahyba do Norte. Serviço Geológico e Mineralógico, Rio de Janeiro. Monographia 8:1-30.

Maury, C.J. 1934. Fossil Inverterbrata from Northeastern Brazil. American Museum of Natural History Bulletin, 67(4):123-179.

Maury, C.J. 1937. O Cretaceo de Sergipe. Serviço Geológico e Mineralógico, Rio de Janeiro, Monographia, 11:1-283.

Néraudeau, D.; Busson, G. \& Cornée, A. 1993. Les Échinides du Cénomanien Supérieur et du Turonien Inférieur du Tinrhert Oriental et Central (Sahara Algérien). Annales de Paleontologie, 79(4):1-313.

Pictet, F. 1857. Traité de Paleontologie ou Histoire naturelle des animaux fossiles considérés dans leurs rapports zoologiques et géologiques. Sécond édition, v. 4, J-B. Ballière, Paris, 768 p.

Pomel, A. 1883. Echinodermes. 1: Classification méthodique et genera dés Échinides vivants et fossiles. Théses présentées à la Faculté des Sciences de Paris pour obtenir le Grade de Docteur és Sciences Naturelles. Première Thèse. Jourdan, Algerie, 151 p.

Simões, M.G. \& Holz, M.2004. Tafonomia: processos e ambientes de fossilização. In: I. de S. Carvalho (ed.) Paleontologia, Editora Interciência, p. 19-37.

Smith, A.B. 1984. Echinoid Palaeobiology. George Allen \& Unwin, London, 199 p.
Smith, A.B. 1995. Late Campanian-Maastrichtian echinoids from the United Arab Emirates-Oman border region. Bulletin of the British Museum Natural History (Geology), 51(2):121-240.

Smith, A.B. 2004. Phylogeny and systematics of holasteroid echinoids and their migration into the deep-sea. Palaeontology, 47(1):123-150.

Smith, A.B. \& Bengtson, P. 1991. Cretaceous echinoids from northeastern Brazil. Fossils and Strata, 31:1-88.

Smith, A.B. \& Jeffery, C.H. 1998. Selectivity of extinction among sea urchins at the end of the Cretaceous period. Nature, 392:69-71.

Souza-Lima, W.; Andrade, E.J.; Bengtson, P. \& Galm. P. 2002. A bacia de Sergipe-Alagoas: evolução geológica, estratigrafia e conteúdo fóssil. Fundação Paleontológica Phoenix, Edição especial, 1, $34 \mathrm{p}$.

Thum, A.B. \& Allen, J. C.1975. Distribution and abundance of the lamp urchin Echinolampas crassa (Bell) 1880 in False Bay, Cape. Transactions of the Royal Society of South Africa, 41:359-373.

Wagner, C.D. \& Durhan, J.M. 1966. Holasteroids. In: R.C. Moore. (ed.) Treatise on Invertebrate Paleontology, Part U Echinodermata. Geological Society of América, University of Kansas , 3, p. U523-U543.

White, C.A. 1887. Contribuições á paleontologia do Brazil. Archivos do Museu Nacional do Rio de Janeiro, 7:1-273.

Received April, 2005; accepted September, 2005. 